Reprod. Nutr. Dévelop., 1988, 28 (1), 101-102.

\title{
Acceptabilité des aliments composés contenant des tourteaux de colza par des tests de préférence sur chèvres
}

\author{
P. MORAND-FEHR, J. HERVIEU
}

Station de Nutrition et Alimentation (I.N.R.A.) de INA.PG 16. rue Claude-Bernard, 75231 Paris Cedex 05.

Summary. Two experiments in goats showed that rapid tests could discriminate the differences of acceptability between rapeseed oilmeals : dehulled or not, treated or not, varieties high $(0)$ and low $(00)$ in glucosinolates. But to get sharp differences, oilmeals must be tested at different contents in compound feeds.

La faible acceptabilité des tourteaux de colza, due notamment à la présence de glucosinolates, est l'un des facteurs limitant leur utilisation dans l'alimentation des ruminants (Beranger et Grenet, 1969). Des tests rapides sur chèvres ont cherché à discriminer des mélanges d'aliments concentrés contenant divers tourteaux de colza selon leur acceptabilité (Morand-Fehr et Hervieu, 1983, Morand-Fehr et al., 1987). Cependant ces tests ont une portée limitée car ils ne concernent qu'un seul taux d'incorporation (TI) et risquent de donner des résultats insuffisants et même erronés. Pour cette raison, nous avons cherché à estimer à différents $\mathrm{TI}$, I'acceptabilité de tourteaux de colza à forte (variété 0) ou faible (variété 00) teneur en glucosinolates, dépelliculés ou non ou ayant subi ou non un traitement technologique.

Matériel et méthodes. Dans une première expérience (Exp. A), 14 chèvres en lactation ont permis de comparer 3 tourteaux de colza ayant subi un toastage de 30 min $\left[C_{1}\right.$ : variété Darmor 00 peu cuit et dépelliculé (dép.); $C_{2}:$ Darmor : 00 fortement toasté et dép. ; $C_{3}$ : Jetneuf : 0 moyennement cuit et non dép.]. Dans une seconde expérience (Exp. B), 4 tourteaux de variété Jetneuf 0 (NN : non traité et non dép., ND : non traité et dép., TN : traité (traitement physique non précisé par le fournisseur) et non dép., TD : traité et dép.) ont été testés sur 12 chèvres. Ces tourteaux ont été incorporés à raison de $10 \%$ (Exp. B seulement), 15, 25 et $45 \%$ à un mélange orge $80 \%$ - Tourteau de soja $20 \%$ ou bien distribués purs.

Chaque jour, deux aliments sous forme granulée (diamètre $4,5 \mathrm{~mm}$ ), sont présentés simultanément à chacune des 14 chèvres qui subissent le test l'une après l'autre. Les chèvres ne participent qu'à un test par jour. Toutes les combinaisons de 2 aliments sont testées au cours de 3 et 6 jours successifs, respectivement dans les exp. A et $\mathrm{B}$. Chaque aliment est présenté dans 2 coupelles contenant $200 \mathrm{~g}$. Dans chaque test, les chèvres sont en présence de 4 coupelies auxquelles elles ont accès pendant 4 intervalles de $20 \mathrm{~s}$, séparés de périodes de fermeture du cornadis de $15 \mathrm{~s}$ pendant lesquelles la place des coupelles est changée. Dans ces conditions, les chèvres consomment au maximum de 550 à $600 \mathrm{~g}$ par test dans le cas de deux aliments de très bonne acceptabilité. Les chèvres reçoivent par ailleurs du foin de luzerne à volonté et un complément d'aliments concentrés calculé pour couvrir leurs besoins. Les tests ont lieu $4 \mathrm{~h}$ après la dernière distribution dans un enclos de $2 \mathrm{~m}^{2}$. Les chèvres sont habituées aux conditions expérimentales de ces tests pendant plusieurs semaines. Les quantités ingérées de chaque aliment sont enregistrées individuellement dans chaque test. Chaque 
aliment est caractérisé par la moyenne des quantités ingérées par toutes les chèvres au cours de tous les tests auxquels il participe.

Résultats et discussion. La figure 1 présente les résultats des expériences $A$ et B. Les aliments contenant $15 \%$ de tourteau dans l'expérience A ou $10 \%$ dans I'expérience $B$ ont des niveaux de consommation comparables quel que soit le type de tourteau. Aucune différence significative n'apparaît sur l'ingestion des tourteaux distribués seuls. C'est à $45 \%$ d'incorporation que les aliments présentent des différences $d^{\prime}$ ingestion les plus importantes.

Les tourteaux 00 dépelliculés semblent posséder une meilleure acceptabilité que le tourteau 0 non dépelliculé mais cette différence n'apparaît nettement quel
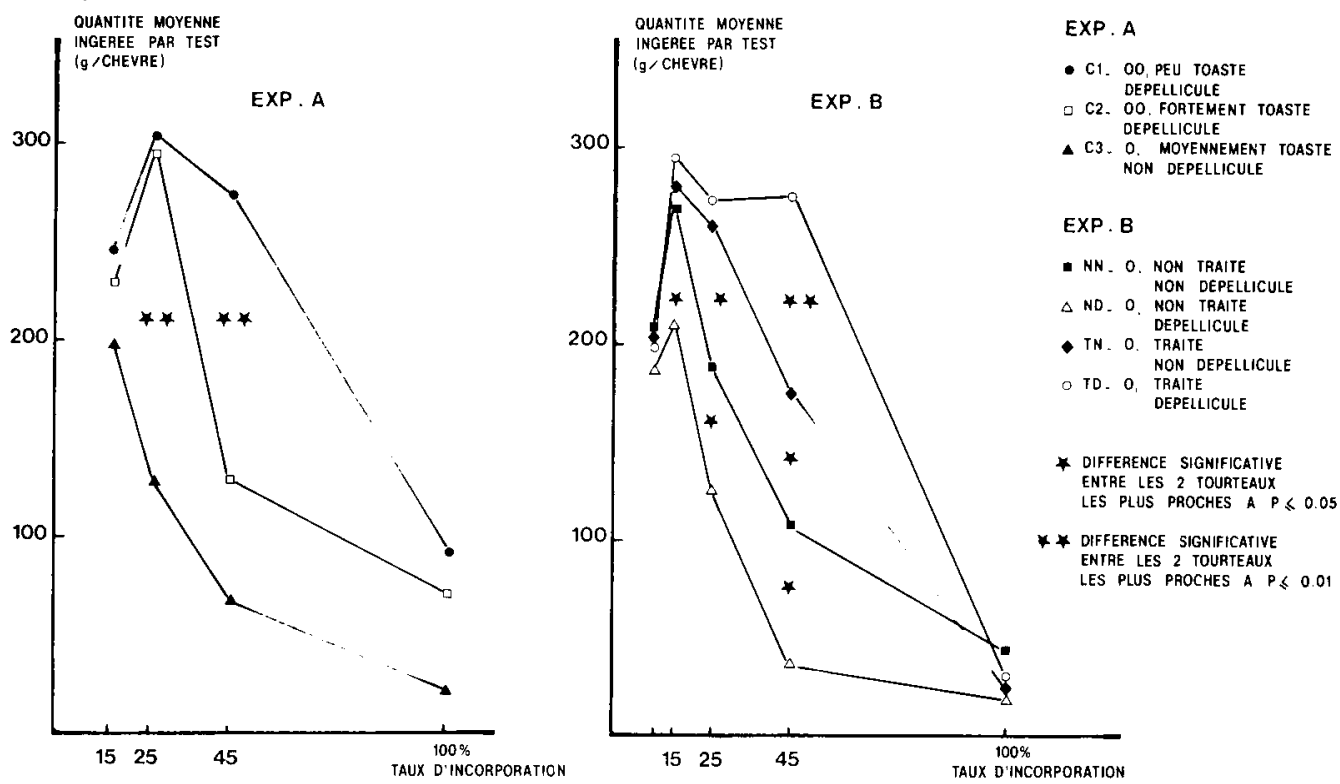

FIG. 1. - Variation de la quantité ingérée par les chèvres des aliments contenant différents tourteaux de colza selon leur taux d'incorporation.

que soit le degré de toastage qu'à $25 \%$ de TI. Toutefois, distribués purs, les tourteaux 00 sont aussi peu consommés que le tourteau 0. Des traitements technologiques des tourteaux 0 et 00 peuvent modifier leur acceptabilité. Le dépelliculage aurait un effet positif sur les tourteaux 0 seulement $s^{\prime}$ ils sont traités, et plutôt négatif s'ils ne sont pas traités. L'ingestion moyenne des animaux tend globalement à être plus faible à $15 \%$ qu'à $25 \%$ (Exp. A) et à $10 \%$ qu'à $15 \%$ de TI (Exp. B). Or, les premiers tests à $15 \%$ (Exp. A) et $10 \%$ de TI (Exp. B) ont été réalisés respectivement après 3 et 2 semaines d'adaptation des chèvres, durée qui pourrait être insuffisante pour obtenir une adaptation satisfaisante.

Conclusion. Ainsi l'acceptabilité des tourteaux de colza dépend fortement de leur origine et de leur traitement. Les chèvres semblent y être très sensibles comme l'avaient montré Morand-Fehr et al. (1983). La discrimination de l'acceptabilité de plusieurs lots de tourteaux de colza semble maximale à un TI de $45 \%$ environ.

Béranger C., Grenet N., 1969. Ann. Zootech., 18, 239-247.

Morand-Fehr P., Hervieu J., 1983. In $6^{e}$ Congr. Int. sur le colza, 2, pp. 1637-1642.

Morand-Fehr P., Hervieu J., Legendre D., Gutter A., Del Tedesco L., 1987. Ann. Zootech., 36, 324 (Abstr.). 